\section{Photothermally induced delayed tissue death}

\author{
Jeffrey M. Gordon, ${ }^{\text {a,b,** Ruthy Shaco-Levy, }}{ }^{c}$ \\ Daniel Feuermann, ${ }^{a}$ Mahmoud Huleihil, ${ }^{a}$ and \\ Solly Mizrahi ${ }^{\text {d }}$ \\ ${ }^{a}$ Ben-Gurion University of the Negev, \\ Jacob Blaustein Institutes for Desert Research, \\ Department of Solar Energy \& Environmental Physics, \\ Sede Boqer Campus 84990, Israel \\ ${ }^{b}$ Ben-Gurion University of the Negev, \\ Pearlstone Center for Aeronautical Engineering Studies, \\ Department of Mechanical Engineering, \\ Beersheva 84105, Israel \\ 'Ben-Gurion University of the Negev, \\ Soroka Medical Center and Faculty of Health Sciences, \\ Department of Pathology, \\ Beersheva 84105, Israel \\ ${ }^{\mathrm{d} B e n-G u r i o n}$ University of the Negev, \\ Soroka Medical Center and Faculty of Health Sciences, \\ Department of Surgery A, \\ Beersheva 84105, Israel
}

Abstract. We report pronounced delayed tissue death in photothermal surgery performed on the livers of live healthy rats with highly concentrated sunlight (ultrabright noncoherent light). Exposure times and power levels were selected to produce immediate necroses of the order of hundreds of cubic millimeters. Pathology reveals that lesion volumes increase by up to a factor of 5 within approximately $24 \mathrm{~h}$ after surgery, and then stabilize. Islands of viable cells can persist within damaged tissue, in the immediate vicinity of blood vessels, but also necrose within about 48 h. () 2006 Society of Photo-Optical Instrumentation Engineers. [DOI: 10.1117/1.2210948]

Keywords: photothermal; solar; necrosis; surgery.

Paper 06015LR received Jan. 28, 2006; revised manuscript received Apr. 26, 2006; accepted for publication Apr. 28, 2006; published online Jun. 9, 2006.

This study began as trials on live healthy animals that demonstrated the effectiveness of highly concentrated sunlight (ultrabright noncoherent light) in producing the same degree, rate, and type of photothermal tissue damage ordinarily achieved with laser fiber-optic surgery, at potentially far lower cost (albeit limited to clear-sky sunny periods). ${ }^{1-3}$ Previously, it had been shown that solar radiation can be concentrated to power densities commensurate with many laser surgery treatments, ${ }^{1,2}$ and can be delivered inside the body via high-transmissivity optical fibers. ${ }^{3}$ A mirrored optic concentrates sunlight into a quartz-core optical fiber with a 1-mm core diameter, a nominal numerical aperture of 0.66 , high transmissivity over the solar spectrum, and extending to a remote indoor operating theater. ${ }^{1-3}$

The optical penetration depth $\delta$ for solar radiation in healthy biological tissue such as liver ranges from the order of tens of microns at the violet end of the spectrum, to several millimetres in the near infrared ${ }^{4,5}$ ( $\delta$ is reduced slightly in coagulated tissue and essentially zero in carbonized tissue). This covers the spectral range of lasers commonly used for

*Tel: 9727659 6923; E-mail: jeff@bgu.ac.il

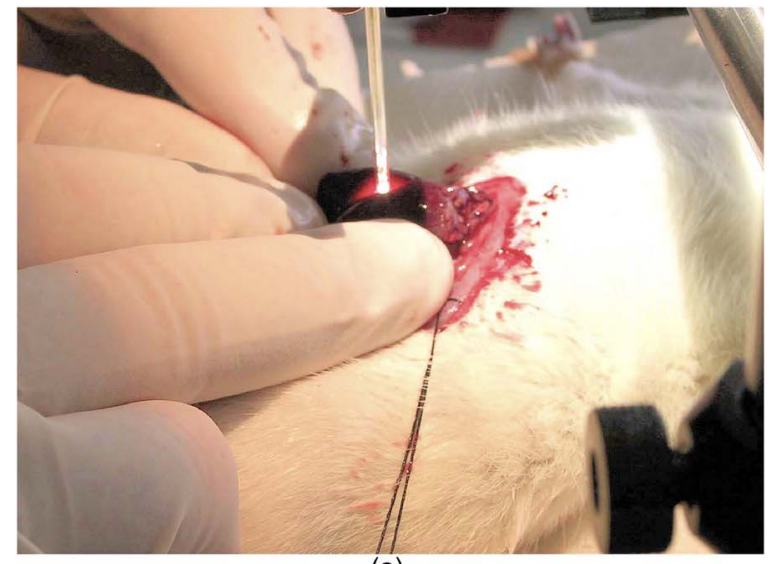

(a)

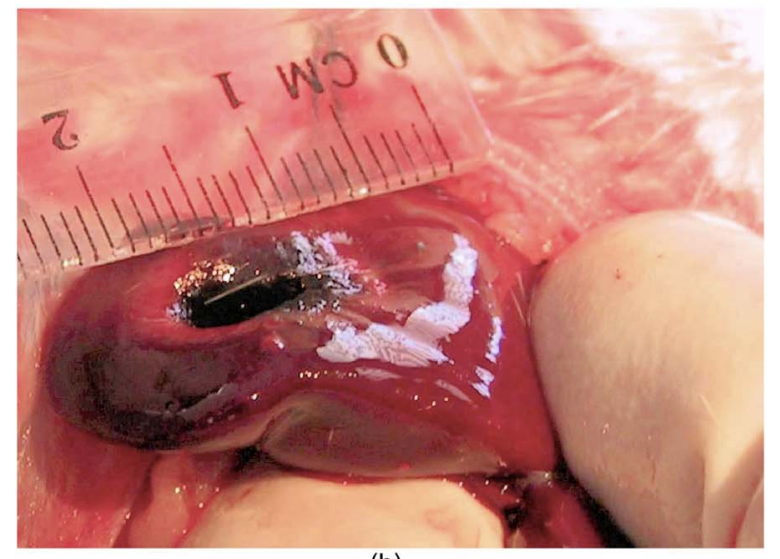

(b)

Fig. 1 Sample of solar surgery on a live anesthetized rat. (a) High-flux solar radiation collected outdoors is transported into the operating theater via an optical fiber, and delivered inside the liver. (b) Lesion photographed immediately after the cessation of irradiation, comprising a dehydrated and carbonized core, encircled by pinkish coagulated tissue (color online only).

interstitial surgery. ${ }^{4-6}$ Hence solar surgery can serve as a probe in exploring the effectiveness and dynamics of lightbased interstitial surgery where intense flux, rather than coherence or monochromaticity, is the essential property. The advantages of light-based coagulative and ablative treatments relative to hot-wire techniques, radiofrequency, and cryogenic ablation have been documented in earlier studies. ${ }^{7}$

Here we report on pronounced tissue death that continues during approximately $24 \mathrm{~h}$ after surgery, and the transient survival of cell conglomerates. Because of the similarity of solar and laser surgery for many photothermal treatments, the phenomena observed here should occur in interstitial laser applications, but, with a few exceptions, ${ }^{8,9}$ appear to have been overlooked.

Since the thermal response time of the tissue is of the order of seconds or minutes, the observed delayed cell death that progresses on a time scale of hours after photothermal surgery is an athermal process. A dramatic thermal insult induces biochemical pathways toward programmed cell death. ${ }^{8,9}$ Delayed tissue death should not be confused with the empirical Arrhenius-like relation that describes the fraction of tissue

1083-3668/2006/11(3)/030504/3/\$22.00 @ 2006 SPIE 
(a)

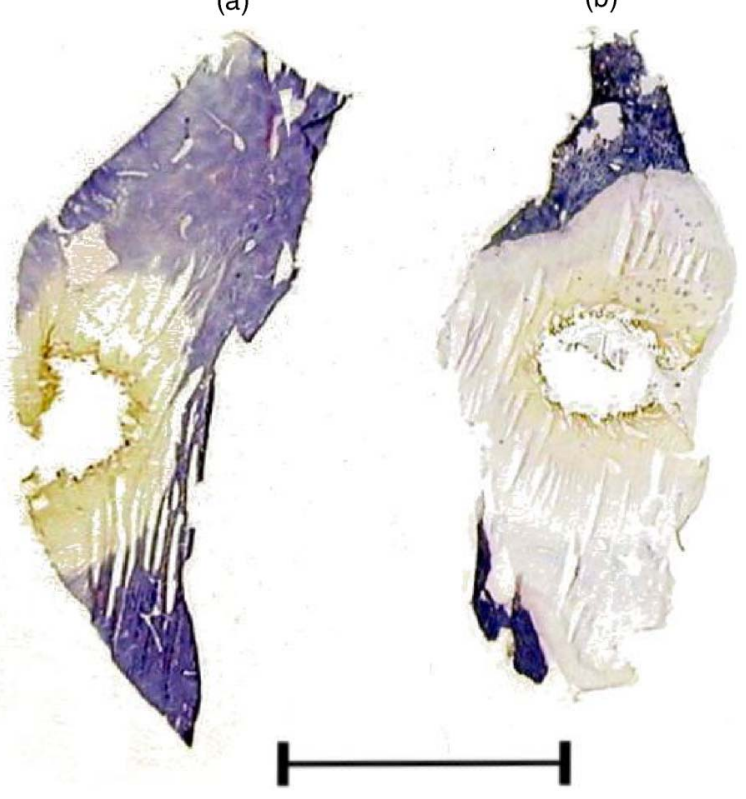

Fig. 2 Pathology samples: transverse sections of liver lesions. Exposure time $=210 \mathrm{~s}$. Viable tissue stains blue (color online only); necrotic regions do not stain. (a) Immediate sacrifice. (b) Sacrifice after $48 \mathrm{~h}$-note the strikingly larger necrosis. Scale bar $=1 \mathrm{~cm}$.

that remains viable upon exposure to a given temperaturetime profile. ${ }^{10}$ Namely, the relatively rapid restoration of tissue temperature to normal (nondamaging) body levels, after exposure to intense light ends, precludes the Arrhenius relation pertaining to more than immediate tissue damage.

The surgical methods and type of animals we employed are the same as reported in our preliminary solar surgery on live rats. ${ }^{3,11}$ Here, we conducted two solar surgery exposures of 120 and $210 \mathrm{~s}$, at an input power of $2.5 \pm 0.2 \mathrm{~W}$, near the center of the thinner and thicker liver lobes, respectively, in 15 animals (Fig. 1). Tissue temperature was not measured. Earlier modeling ${ }^{2,10}$ for comparable radiative power input and exposure times would indicate that, at the cessation of irradiation, tissue temperature is distributed in a strongly nonlinear fashion, from as high as $\sim 300^{\circ} \mathrm{C}$ at the center of the lesion, to $\sim 60^{\circ} \mathrm{C}$ at the rim of the region perceived as coagulated. From that rim, the temperature decreases over a surrounding volume far larger than the immediate necrosis to $\sim 37^{\circ} \mathrm{C}$ at the healthy unaffected surrounding liver tissue.

The radiative power level and exposure times were chosen to generate immediate necroses of the order of hundreds of cubic millimeters: a compromise between the desire to demonstrate lesion volumes typically required in surgery on humans, yet accounting for the vastly smaller liver size in rats where we measured midlobe thickness in the range 5 to $12 \mathrm{~mm}$.

Rats were sacrificed at 0,24 , and $48 \mathrm{~h}$ after surgery. For histopathology examination, we adopted NADH-diaphorase (NADH), rather than the standard hematoxylin and eosin (H\&E) stain. Recently, we showed that the H\&E method can yield order-of-magnitude underestimates of tissue death when pathology is performed immediately after surgery ${ }^{11}$ (although the shortfall is mitigated when histological examination is postponed for $24 \mathrm{~h}$ ). In contrast, NADH provided consistently
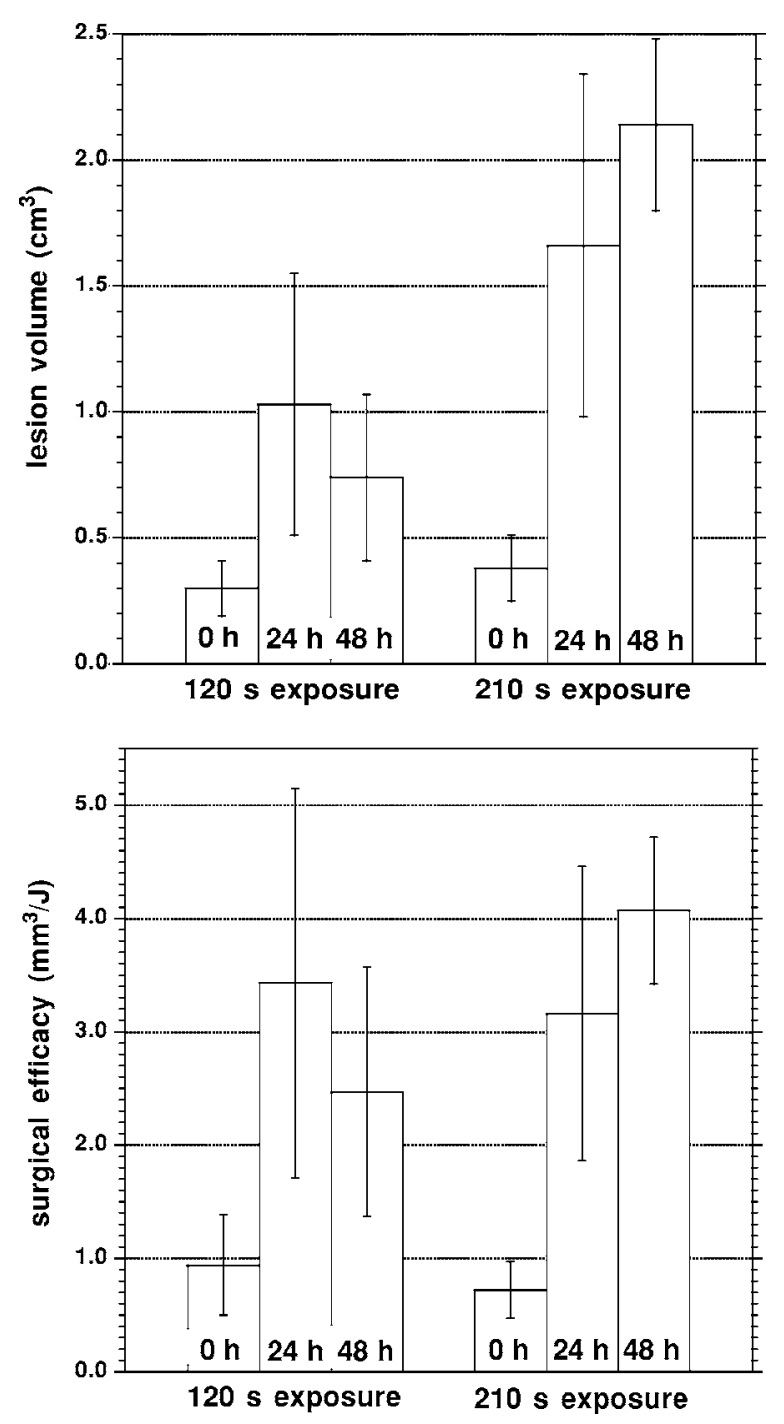

Fig. 3 Summary of lesion volume and surgical efficacy for sacrifice at 0,24 , and $48 \mathrm{~h}$, and solar surgery exposure times of 120 and $210 \mathrm{~s}$.

accurate results at all examination times considered here.

Necrotic zones varied in shape from roughly hemispherical to spherical, depending on how deeply the fiber tip was inserted into the liver. The sample transverse sections in Fig. 2 illustrate the pronounced difference in lesion dimensions for immediate and delayed lesions. All sections exhibited the same signature tissue transformations: a central area of carbonization and tissue dehydration, surrounded by a small rim with features of coagulative necrosis. Quantitative results are tabulated in Fig. 3.

Instances where the immediate lesion traversed the entire lobe (primarily the thinner lobes) are equally represented in Fig. 3, but constitute a lower bound for lesion size and surgical efficacy (surgical efficacy denoting lesion volume per unit of delivered radiative energy). This contributes to the relatively large variance in lesion size (and hence in surgical efficacy) for nominally identical surgical conditions. The same qualification pertains to lesions that covered nearly the full lobe thickness when surgery was terminated. Their depth reached the lobe thickness prior to the end of delayed necrosis. The largest lesions $\left(2.5 \mathrm{~cm}^{3}\right)$ and highest surgical efficacy 


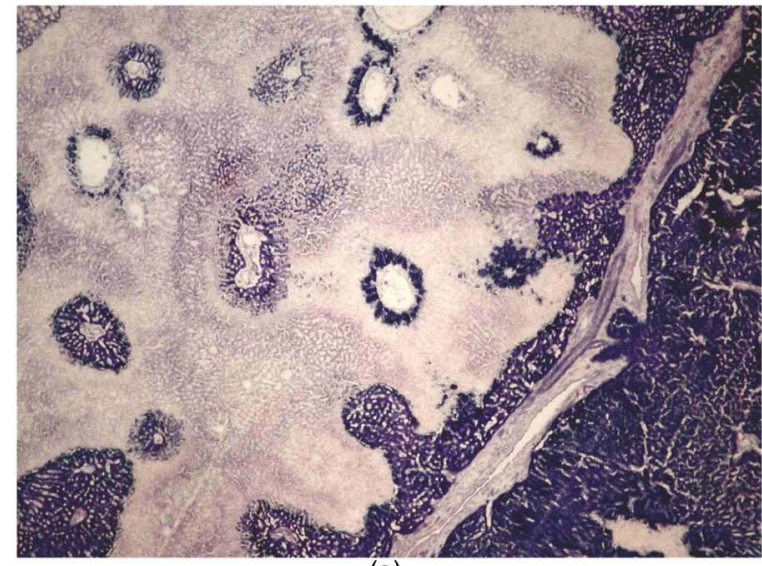

(a)

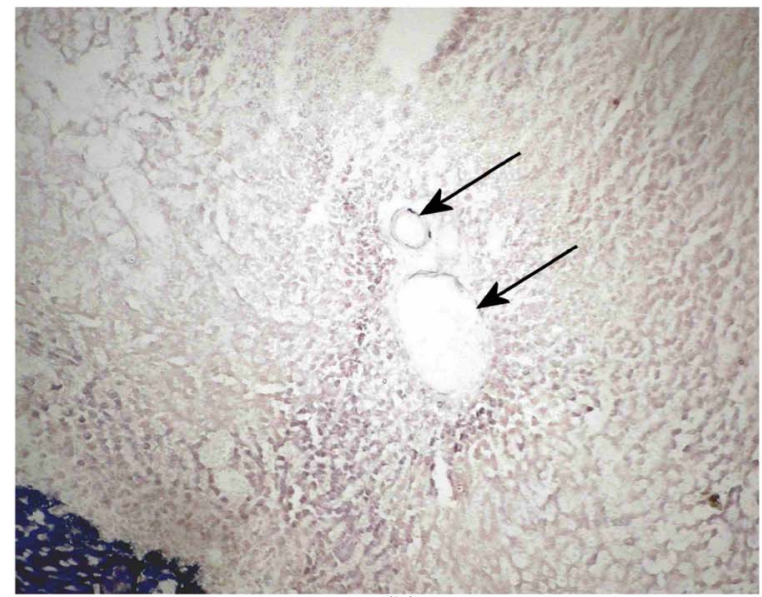

(b)

Fig. 4 Pathology slides illustrating "islanding." (a) Sacrifice after 24 h, with clearly discernible regions of viable cells (dark stain) having survived in the neighborhood of blood vessels within a necrosed zone (dead cells do not stain). (b) Sacrifice after $48 \mathrm{~h}$, at which stage all viable cell islands have succumbed to necrosis. Arrows indicate the periphery of two blood vessels.

values (4 to $6 \mathrm{~mm}^{3} / \mathrm{J}$ ) correspond to the thickest livers. Here, as in earlier reports, ${ }^{3,11}$ the efficacy with solar surgery was found to be comparable to that for corresponding laser fiberoptic procedures. $^{12,13}$

Figure 4 reveals an "islanding" phenomenon. The lesions in animals sacrificed immediately were devoid of surviving tissue. However, in about half the lesions in animals sacrificed after $24 \mathrm{~h}$, small regions of viable tissue, in the immediate vicinity of blood vessels, survived within a sea of necrotic cells. The viable islands always yielded to necrosis within $48 \mathrm{~h}$.

The chief discovery in these trials was massive delayed cell death that continues for about $24 \mathrm{~h}$ after surgery, in a nonthermal process (ignited by a thermal insult) where lesion volume increases by up to a factor of 5. Postoperative necrosis can proceed in the immediate vicinity of blood vessels, with transient islands of viable cells that die within 24 to $48 \mathrm{~h}$. We stress, however, that our observations in this work (as well as those of Refs. 8 and 9) relate only to liver tissue.

The relevance of the findings lies in (1) allowing projections of eventual necrosis during surgery, toward avoiding unwarranted damage to untargeted tissue, and (2) minimizing the danger of false positives from the large tissue volume that appears viable during surgery but will experience delayed necrosis within $24 \mathrm{~h}$. Deciphering the biochemical basis for such delayed tissue death remains a challenge. ${ }^{8,9}$ The objective here has been documenting the phenomenon and its dynamics, with emphasis on the enormity of delayed tissue death when induced with high photonic flux.

\section{Acknowledgments}

This work was supported by a grant from the Rita Altura Foundation, Los Angeles, CA. We are grateful to Rafi Itah for surgical assistance, and thank Yona Lichtenfeld and Lubov Stefanovsky for technical assistance in pathology slide preparation. This study was approved by the Institutional Animal Care and Use Committee of the Soroka Medical Center, BenGurion University, Beersheva, Israel, and the surgeon (S.M.) underwent the health protection program for handling research animals.

\section{References}

1. J. M. Gordon, D. Feuermann, and M. Huleihil, "Laser surgical effects with concentrated solar radiation," Appl. Phys. Lett. 81, 2653-2655 (2002).

2. J. M. Gordon, D. Feuermann, M. Huleihil, S. Mizrahi, and R. ShacoLevy, "Solar surgery," J. Appl. Phys. 93, 4843-4851 (2003).

3. J. M. Gordon, D. Feuermann, M. Huleihil, S. Mizrahi, and R. ShacoLevy, "Surgery by sunlight on live animals," Nature (London) 424, 510 (2003).

4. A. Katzir, Lasers and Optical Fibers in Medicine, Academic Press, San Diego (1993).

5. A. J. Welch and M. J. C. van Gemert, Optical-thermal Response of Laser-irradiated Tissue, Plenum Press, NY (1995).

6. R. M. Verdaasdonk and F. P. van Swol, "Laser light delivery systems for medical applications," Phys. Med. Biol. 42, 869-894 (1997).

7. J. T. De Sanctis, S. N. Goldberg, and P. R. Mueller, "Percutaneous treatment of hepatic neoplasms: a review of current techniques," Cardiovasc. Intervent Radiol. 21, 273-296 (1998).

8. Y. Fujitomi, K. Kashima, S. Ueda, Y. Yamada, H. Mori, and Y. Uchida, "Histopathological features of liver damage induced by laser ablation in rabbits," Lasers Surg. Med. 24, 14-23 (1999).

9. M. Nikfarjam, V. Muralidharan, and C. Christophi, "Mechanisms of focal heat destruction of liver tumors," J. Surg. Res. 127, 208-223 (2005).

10. M. N. Iizuka, I. A. Vitkin, M. C. Kolios, and M. D. Sherar, "The effects of dynamical optical properties during interstitial laser photocoagulation," Phys. Med. Biol. 45, 1335-1357 (2000).

11. R. Shaco-Levy, J. M. Gordon, S. Mizrahi, D. Feuermann, and M. Huleihil, "On appropriate pathology for photothermal surgery," Lasers Surg. Med. 35, 28-34 (2004).

12. J. Heisterkamp, R. van Hillegersberg, E. Sinofsky, and J. N. M. Ijzermans, "Heat-resistant cylindrical diffuser for interstitial laser coagulation: comparison with the bare-tip fiber in a porcine liver model," Lasers Surg. Med. 20, 304-309 (1998).

13. D. Albrecht, C. T. Germer, C. Isbert, J. P. Ritz, A. Roggan, G. Müller, and H. J. Buhr, "Interstitial laser coagulation: evaluation of the effect of normal liver blood perfusion and the application mode on lesion size," Lasers Surg. Med. 23, 40-47 (1998). 